\title{
Femtosecond X-ray Absorption Spectroscopy of a Light-Driven Spin-Crossover Process
}

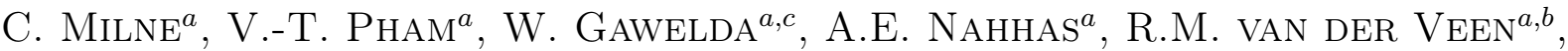

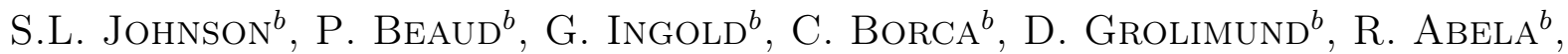 \\ M. Chergui ${ }^{a}$ And Ch. Bressler ${ }^{c, *}$
}

${ }^{a}$ Laboratoire de Spectroscopie Ultrarapide (LSU), Ecole Polytechnique Fédérale de Lausanne (EPFL), BSP, CH-1015 Lausanne, Switzerland

${ }^{b}$ Swiss Light Source, Paul Scherrer Institut, CH-5232 Villigen-PSI, Switzerland

${ }^{c}$ European XFEL GmbH, c/o DESY, Notkestrasse 85, D-22607 Hamburg, Germany

\begin{abstract}
Understanding the initial steps during ultrafast molecular reactions involving large spin state changes is a vital goal in structural dynamics research. These involve knowledge of the geometric structure of the system. Ultrafast X-ray absorption spectroscopy with 50-100 picosecond time resolution establishes the geometric structure of the short-lived $(\tau=0.6 \mathrm{~ns})$ high spin state. Here we focus on time-resolved X-ray absorption studies with 160-200 fs temporal resolution to monitor the structural evolution in this spin-conversion process.
\end{abstract}

PACS numbers: 78.70.Dm, 75.30.Wx

\section{Introduction}

Time-resolved X-ray absorption fine structure (XAFS) spectroscopy with picosecond temporal resolution has recently been established as a new method to observe electronic and geometric structures of short-lived reaction intermediates in solution $[1,2]$. It combines an intense femtosecond laser source synchronized to the 50-100 ps $\mathrm{X}$-ray pulses delivered at the microXAS beamline of the Swiss Light Source (SLS). To go to the sub-picosecond time resolution, femtosecond X-ray pulses can be extracted via the time-slicing scheme $[3,4]$. We used this scheme in the hard X-ray domain at the microXAS beamline (SLS), to investigate the light-induced magnetization of aqueous Iron(II)-tris $\left(2,2^{\prime}\right)$-bipyridine $\left(\left[\mathrm{Fe}^{\mathrm{II}}(\mathrm{bpy})_{3}\right]^{2+}\right)$, which is completed within $300 \mathrm{fs}$ [5]. This is the first example of a femtosecond hard X-ray absorption experiment of a molecule in liquids.

\section{Light-driven spin crossover at room temperature in solution}

$\left[\mathrm{Fe}^{\mathrm{II}}(\mathrm{bpy})_{3}\right]^{2+}$ represents the simplest molecule of iron-based light-induced spin-cross over complexes, itself being a typical example of a low spin (LS) compound, which can undergo a spin change to a quintet state upon irradiation [6]. The optical absorption spectrum of aqueous

* corresponding author; e-mail: christian.bressler@xfel.eu
$\left[\mathrm{Fe}^{\mathrm{II}}(\text { bpy })_{3}\right]^{2+}$ is characterized by an intense broad band centred at $520 \mathrm{~nm}$ due to the singlet Metal-to-Ligand-Charge-Transfer $\left({ }^{1} \mathrm{MLCT}\right)$ state. Photoexcitation into this band (or to higher energies) is followed by a cascade of intersystem crossing (ISC) steps through singlet, triplet and quintet MLCT and ligand-field (LF) states, which brings the system to the lowest-lying (HS) quintet state, ${ }^{5} T_{2}$, with almost unit quantum yield in $<1 \mathrm{ps}$ [7]. This state relaxes non-radiatively to the LS ground state within $0.6 \mathrm{~ns}$ in aqueous solutions at room temperature. Using picosecond XAS, we recently determined that in the HS state, an elongation of $\sim 0.2 \AA$ of the $\mathrm{Fe}-\mathrm{N}$ bond distances occurs [2].

However, the pathway and time scale of the cascade from the ${ }^{1} \mathrm{MLCT}$ to the ${ }^{5} T_{2}$ state are still not known, as ultrafast optical spectroscopy can neither resolve the intermediate steps nor determine their structures. In order to address these issues and to probe the relaxation processes, we have implemented femtosecond XANES spectroscopy.

\section{Femtosecond X-ray absorption spectroscopy}

Tunable hard X-ray pulses with 140 (30) fs pulse width in the $5-20 \mathrm{keV}$ range are extracted via the time-slicing scheme at the microXAS beamline, as described in Ref. [4]. We performed the experiments at the iron $K$ edge around $7 \mathrm{keV}$ with approximately 10 photons/pulse onto the sample at $2 \mathrm{kHz}$ repetition rate within a bandwidth of approximately $2 \mathrm{eV}$. The instrument response 
width, given by the cross correlation between the X-ray and the laser pulse widths, is 160-200 fs in agreement with jitter and long term (days) drift measurements [4]. Time delay scans at a fixed energy were recorded, next to energy scans at a fixed time delay. The precise time zero (and thus the selected time delay) was quantified from the data after fitting the data to a rate model (see below).
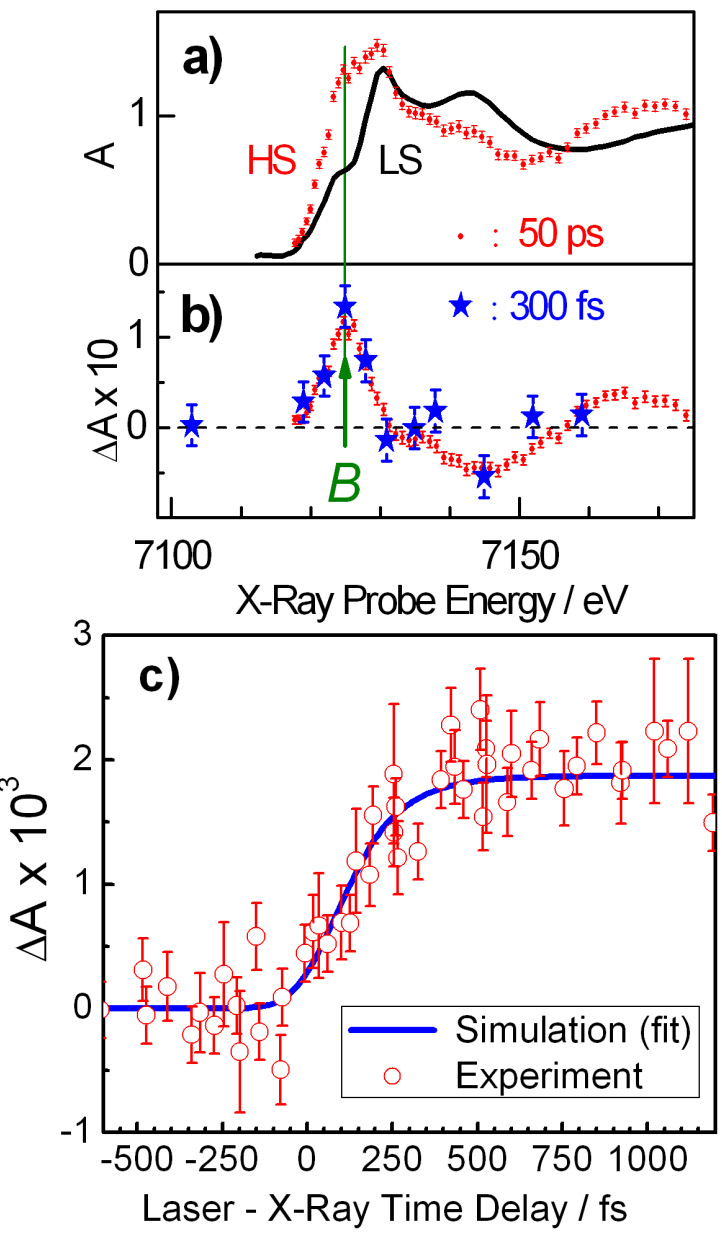

Fig. 1. (a) Picosecond X-ray absorption near-edge structure spectra of $\left[\mathrm{Fe}(\mathrm{bpy})_{3}\right]^{2}$ in its low-spin state (LS, black curve) and high-spin (HS, data points) excited state; (b) transient X-ray absorption spectrum of photoexcited aqueous $\left[\mathrm{Fe}(\mathrm{bpy})_{3}\right]^{2+}$ after 50 ps (circle data points) and after $300 \mathrm{fs}$ (stars with error bars); (c) femtosecond time trace of the evolution of the strongest transient X-ray absorption feature in (b) (near $7125 \mathrm{eV}$ ) together with a fit according to reaction sequence via Eq. (1).

\section{Results and discussion}

Figure 1a shows the LS (ground state) spectrum together with the extracted HS (excited state) spectrum, and Fig. 1b displays the transient absorption spectrum after 50 ps [2], together with the transient XANES measured after 300 fs. The strongest absorption change occurs at a multiple scattering edge feature near $7126 \mathrm{eV}$ and reflects the increased $\mathrm{Fe}-\mathrm{N}$ bond distance [5], determined to be $0.2 \AA[2,8]$. We therefore used this feature to investigate the temporal evolution of the relaxation process from the ${ }^{1} \mathrm{MLCT}$ to the ${ }^{5} T_{2}$ by scanning the laser$\mathrm{X}$-ray time delay, which also confirms that this process terminates below 300 fs (Fig. 1b). In order to quantify the time required for this spin crossover process, we have calculated the rate equations for this process. Hereby, the following reaction cycle, using the input from our optical studies [7], was applied (using the indicated lifetimes and an overall cross correlation time of $200 \mathrm{fs}$ [4]):

$$
\begin{aligned}
& { }^{1} \mathrm{GS}+h \nu(400 \mathrm{~nm}) \rightarrow{ }^{1} \operatorname{MLCT}(20 \mathrm{fs}) \\
& \quad \rightarrow{ }^{3} \operatorname{MLCT}(120 \mathrm{fs}) \rightarrow{ }^{5} \mathrm{~T}(665 \mathrm{ps}) \rightarrow{ }^{1} \mathrm{GS}
\end{aligned}
$$

With this scenario we calculated the population dynamics of all intermediate states (as given above), and determined the final HS signal, which is shown in Fig. 1c together with the data. In addition, we fit the final arrival time to the ${ }^{5} \mathrm{~T}$ state (previously fixed to the ${ }^{3} \mathrm{MLCT}$ departure time of $120 \mathrm{fs}$ ) yielding 130 (60) fs. This result implies that the electron back transfer from the ligand system simultaneously triggers the excitation of a second electron from the bonding $t_{2 \mathrm{~g}}$ orbital, so that both electron spins are parallel in the antibonding $e_{\mathrm{g}}$ orbital, together with two unpaired electrons from the bonding $t_{2 \mathrm{~g}}$ orbitals. Any possible intermediate steps (in the metal-centered states) are considerably faster than $60 \mathrm{fs}$.

\section{Conclusions and outlook}

Via femtosecond X-ray absorption spectroscopy we determined the spin-crossover process to occur within $130 \mathrm{fs}$, which is identical to the electron back transfer time from the ligand system to the metal-centered orbitals. This represents the first direct measurement of the ${ }^{5} T$ arrival time. Optical methods so far failed to observe this rise time, as this state remains optically silent throughout the visible/IR range. While the XAFS experiment determines the $\mathrm{Fe}-\mathrm{N}$ distance to increase by $0.2 \AA$ with the 130 fs population time, it does not measure vibrational relaxation within the HS state due to the poor cross correlation time, but only the ensemble and time averaged distance change. Nevertheless, the quantitative analysis reveals that the system has undergone a prompt ISC step ${ }^{3} \mathrm{MLCT} \rightarrow{ }^{5} \mathrm{~T}$ involving two electrons simultaneously, thus directly coupling both states and bypassing intermediate (single electron) steps. We are currently investigating the anticipated vibrational cooling (of about $1.5 \mathrm{eV}$ ) within the promptly populated hot ${ }^{5} \mathrm{~T}$ state.

This experiment exploiting femtosecond hard Xradiation is a promising approach to add a new observable for our understanding of spin-dynamics in molecular systems. With this and new intense femtosecond X-ray sources becoming available (e.g., XFELs) it is possible to apply this method to more complex systems, e.g., 
to study ISC processes in biological systems, including heme-proteins.

\section{Acknowledgments}

This work was funded by the Swiss National Science Foundation (FNRS) via contracts: 116023, 110464, 107956, 620-066145, and 105239; and by the SBF via contract COST D35 C06.0016.

\section{References}

[1] C. Bressler, M. Chergui, Chem. Rev. 104, 1781 (2004).

[2] W. Gawelda, V.-T. Pham, M. Benfatto, Y. Zaushitsyn, M. Kaiser, D. Grolimund, S.L. Johnson, R. Abela, A. Hauser, C. Bressler, M. Chergui, Phys. Rev. Lett. 98, 057401 (2007).

[3] R.W. Schoenlein, S. Chattopadhyay, H.H.W. Chong, T.E. Glover, P.A. Heimann, C.V. Shank, A.A. Zholents, M.S. Zolotorev, Science 287, 5461 (2000).
[4] P. Beaud, S.L. Johnson, A. Streun, R. Abela, D. Abramsohn, D. Grolimund, F. Krasniqi, T. Schmidt, V. Schlott, G. Ingold, Phys. Rev. Lett. 99, 174801 (2007).

[5] Ch. Bressler, C. Milne, V.T. Pham, A. ElNahhas, R.M. van der Veen, W. Gawelda, S. Johnson, P. Beaud, D. Grolimund, C.N. Borca, G. Ingold, R. Abela, M. Chergui, Science 323, 489 (2009).

[6] A. Hauser, C. Enachescu, M.L. Daku, A. Vargas, N. Amstutz, Coord. Chem. Rev. 250, 1642 (2006).

[7] W. Gawelda, A. Cannizzo, V.-T. Pham, F. Van, C. Mourik, M. Bressler, Chergui, J. Am. Chem. Soc. 129, 8199 (2007).

[8] W. Gawelda, V.-T. Pham, R.M. van der Veen, D. Grolimund, R. Abela, M. Chergui, C. Bressler, J. Chem. Phys. 130, 124520 (2009). 